\title{
Adolescents' Postural Control Learning According to the Frequency of Knowledge of Process
}

\author{
Julián Gandía, Xavier García-Masso, Adrián Marco-Ahullo, and \\ Isaac Estevan \\ University of Valencia
}

\begin{abstract}
Feedback is one of the most influential factors for motor skills learning. Physical Education teachers commonly use verbal cues to provide knowledge of process (KP) when teaching motor skills, but the ideal presentation frequency for KP in adolescents is unclear. The aim of this study was to compare the effectiveness of the frequency of KP (i.e., 100\%, 67\%, 0\%) on dynamic balance. Thirty adolescents, age 14-15 years, participated in the study. Performance on a stabilometer platform was used to assess dynamic balance. Participants received feedback after each trial (100\%), in two out of three trials (67\%), or no feedback during 1230 -s trials of practice. Adolescents who received feedback $(67 \%$ or $100 \%)$ required lower mean velocity to maintain similar dynamic balance performance (i.e., root mean square). Moreover, adolescents receiving 100\% feedback had a higher $\alpha$-scaling than those who did not received it. During the post-test and the retention, both $67 \%$ and $100 \% \mathrm{KP}$ frequencies were effective at improving postural control, compared to the no feedback control.
\end{abstract}

Keywords: augmented feedback, dynamic balance, motor learning, verbal cues

Motor learning is a process whereby new motor skills are acquired (Fujii, Lulic, \& Chen, 2016) with changes in motor performance caused as a result of practice (Sigrist, Rauter, Riener, \& Wolf, 2013). Factors that affect motor learning include, but are not limited to, the task demands, the test condition, and the level of challenge emerging from an interaction with the information-processing capability of the learner (Guadagnoli \& Lee, 2004). Moreover, the feedback received by the learners during or after each practice trial or group of practice trials (Schmidt \& Wrisberg, 2008) can also influence the process of motor skills learning (Fujii et al., 2016; Wulf, Chiviacowsky, Schiller, \& Avila, 2010).

Gandía, García-Massó, Marco-Ahulló, and Estevan are with the Department of Teaching Music, Visual and Corporal Expression; García-Massó is also with the Human Movement Analysis Group; Estevan is also with the AFIPS Research Group; University of Valencia, Valencia, Spain. Estevan (isaac.estevan@ uv.es) is corresponding author. 
Feedback can be classified according to the instant at which it is provided: concurrent and terminal feedback are reported during or after motor task execution, respectively (Sigrist et al., 2013). Terminal feedback facilitates learning as soon as the learner is able to associate this reported feedback with the prior performance (Sülzenbrück \& Heuer, 2011). Both concurrent and terminal feedback can be reported according to the observed source of the information: intrinsic or augmented feedback. On the one hand, intrinsic feedback refers to intrinsic sensory information resulting from movement including vision or proprioception from limbs (Fujii et al., 2016). On the other hand, augmented feedback, which can be seen as supplemental information to intrinsic feedback, is an external source of information given to the learner to emphasize certain aspects of the movement that should be monitored (Schmidt \& Lee, 2011). Augmented feedback has been reported to improve motor skill learning effectiveness (Sigrist et al., 2013) because it facilitates the achievement of the objectives of the skill and motivates learners to continue pursuing to achieve the movement goal (Schmidt \& Wrisberg, 2008). Augmented feedback can be provided via most sensory systems including haptic, audition or vision (Sigrist et al., 2013). While, augmented feedback is often provided via technical displays such as screens, head-mounted displays, speakers, headphones, robotics vibro-tactile actuators or a combination of them (Sigrist et al., 2013), it can also be provided verbally (Schmidt \& Wrisberg, 2008). This is especially important for educators such as Physical Education (PE) teachers who provide verbal augmented feedback to influence the students' motor skill learning.

Augmented feedback most frequently transmitted takes on one of two forms: knowledge of results (KR) and knowledge of performance or process (KP) (Fujii et al., 2016). On the one hand, $\mathrm{KR}$ is known as the information related to the achievement of a movement goal (Magill, 2001). KR can be useful when students need information to determine the outcome of an action or to motivate learners to continue practicing (Magill, 2001). On the other hand, KP is related to the characteristics of the specific components of the movement (Magill, 1998), that is, the nature of the movement pattern that realizes a particular performance outcome (Fujii et al., 2016). Sharma, Chevidikunnan, Khan, and Gaowgzeh (2016) found that both KR and KP were effective for enhancing skilled motor learning, however KP leads to a better motor skill acquisition than the provision of KR. Reporting KP verbally can be beneficial when a specific component of a skill that requires complex coordination must be improved or corrected (Magill, 2001). However, the limited evidence available is not sufficient to justify including terminal-augmented feedback in PE curricula (Sattelmayer, Elsig, Hilfiker \& Baer, 2016).

During PE lessons, verbal cues used by PE teachers when teaching motor skills seems to be useful for guiding students to attend to the critical elements of skill execution and highlighting the most common mistakes they may encounter (Schmidt \& Lee, 2011). Effective verbal cues may reduce the cognitive load necessary to process relevant information from the execution of skills and to focus attention on critical sensory information (Landin, 1994). Effective verbal cues should be characterized as such: 1) reduced to a few concise words or phrases (Perreault \& French, 2016); 2) accurate, critical to the intended task, limited in number; and 3) adjusted to student's age and skill level (Rink, 2006). With regard to age, the ability in cognitive processes such as selective attention and the speed of information processing increases as children age (Ferguson \& Bowey, 2005). 
Therefore, motor learning principles for adults and children cannot be generalized because differences in cognitive abilities may contribute to differences in motor learning (Pollock \& Lee, 1997; Sullivan, Kantak, \& Burtner, 2008).

Differences between children, adolescents, and adults is an important consideration when contemplating what might be an optimal feedback frequency that facilitates motor learning in simple or complex motor tasks. Findings from studies conducted with adults addressing the frequency of augmented feedback for facilitating motor learning in simple tasks (Wulf \& Shea, 2004) are consistent with the guidance hypothesis that postulates an excess of feedback during acquisition is detrimental to the motor skill learning (Schmidt, Young, Swinnen, \& Shapiro, 1989). The guidance hypothesis assumes it is better to give less frequent augmented feedback because the students can use their own inherent feedback to learn the skill (Fujii et al., 2016), allowing them to become relatively independent of the augmented feedback (Wulf et al., 2010). However, children who received less frequent augmented feedback during the practice of a simple task exhibited lower motor learning than those who received $100 \%$ feedback (Wulf et al., 2010). It is possible that when feedback is reported after every practice trial of a simple task, it is more effective than when feedback is progressively reduced (Sullivan et al., 2008). Hence, it seems that to optimize motor learning in simple tasks, in children, it can be effective to provide feedback for every trial. When complex tasks are performed (i.e., those characterized by a high amount of degrees of freedom, conducted in an ecological environment, and featuring a task that cannot be mastered in a single session [Wulf \& Shea, 2002]) by young adults, in contrast with those who received less frequent or no feedback, those who received feedback after every trial exhibited a clear performance improvement (Wulf, Shea, \& Matschiner, 1998). However, there is a lack of research in children and adolescents addressing the frequency of augmented verbal feedback, specifically KP, on motor skills learning during complex tasks. As a result, it is not clear that in relative complex motor tasks (i.e., a task accomplishing one or two of the three characteristics of a complex task) the guidance hypothesis is valid in these individuals.

The aim of this study was to evaluate the effect of different frequencies of verbal-terminal-augmented feedback KP during acquisition and retention on a dynamic balance task in adolescents. Because Sullivan et al. (2008) found that children's motor learning was greater when they received $100 \%$ rather than $62 \%$ feedback when learning a simple task, it is hypothesized that, for the relatively complex balance task it would be necessary to provide feedback to adolescents after every trial rather than less frequently (Wulf et al., 2010).

\section{Method}

\section{Study Design}

The study was used to determine the effect of different frequencies of verbalterminal-augmented feedback KP on dynamic balance in adolescents. Participants were randomly assigned to one of three equal-sized groups (10 participants) that received $100 \%$ feedback, $67 \%$ feedback, or no feedback during practice. These different feedback frequencies are consistent with those used in previous studies in the motor learning field involving both children and adults (Sullivan et al., 2008; 
Wishart \& Lee, 1997; Wulf \& Schmidt, 1989). A pre-test, practice, and post-test were performed by all participants. Furthermore, 24 hours after the completion of practice, the subjects performed a retention test. The Institutional Review Board of the University of Valencia approved the study.

\section{Participants}

A sample size calculation was performed using the data reported by Wulf, Weigelt, Poulter, and McNevin (2003) to calculate the effect size (i.e., $\omega^{2}=0.21$ ). Using $\mathrm{G}^{*}$ Power 3.1 software (University of Düsseldorf, Düsseldorf, Germany), setting the $\alpha$-level at 0.05 and the statistical power at 0.8 , the sample size needed per group was 9 participants. Thus, 30 normal-developed adolescents ( 23 boys and 7 girls) mainly aged 14-15 years participated in the study.

\section{Procedure}

Each participant performed 1230 -s trials on a dynamic balance task. The number of trials was determined as an appropriate number for motor learning in a balance task without introducing fatigue (Thomas, Cotten, Spieth, \& Abraham, 1975). Verbalterminal-augmented feedback was provided with different frequencies of KP (i.e., $100 \%, 67 \%, 0 \%$ ). Before, after, and 24 hours after the practice, participants performed the pre-test, post-test, and retention, respectively, which consisted of three 30-s trials. A rest period of between 30-60 s was allowed between trials.

Instruments. A stabilometer was used that consisted of a wooden platform $(60 \times 40 \mathrm{~cm})$ with a central axis that allowed medio-lateral rotations with a maximum possible deviation of the platform to either side of 15 degrees. On top of one, a Wii Balance Board (WBB) was placed to collect the data during the pre-test, practice, post-test, and retention trials. The platform was placed on the ground at a distance of 2 meters from the wall. A target point was located on the wall at the height of each student's eyes. The objective of the test was to maintain balance defined as keeping the platform in a horizontal position for as long as possible during each 30-s trial.

Protocol. Prior to participation, the PE teacher provided a verbal description and demonstration of the skill (i.e., dynamic balance task). The participants had to keep the platform as horizontal as possible during every trial. To start each trial, the stabilometer platform had to be in contact with the floor on the dominant side of participants. The position of the bare feet on the WBB was parallel and separated by the same distance as an individual's shoulder width during all the trials. The data collection started $1 \mathrm{~s}$ after the receipt of the start signal.

Depending on experimental group assignment, the participants received a predetermined frequency of verbal-terminal-augmented feedback. The $100 \%$ feedback group received feedback after each trial, while the $67 \%$ feedback group received feedback for two out of every three trials. A non-feedback group (0\%) was also assigned as the control group. Feedback was provided only during the practice trials. Participants were given verbal-terminal-augmented feedback once they had completed the trial and had left the platform. Five verbal cues were provided to participants based on five items according to norm balance data in children 
(Condon \& Cremin, 2014; Villarrasa-Sapiña et al., 2016) when corresponded: 1) look at the point placed in front at all times (i.e., remember to look at the point in front of you during the whole trial); 2) compensation strategies with the arms and shoulders (i.e., you can use your arms to rebalance yourself by putting them at the height of the shoulders); 3) rebalancing strategies with the hip and knee (i.e., you can use knee and hip flexion-extension to balance your body); 4) pressure-force strategies of both feet (i.e., feel the pressure in your feet and try to avoid sudden movements); 5) attitude and silence (i.e., remember to be quiet and focus on your balance during the trial).

\section{Data Analyses}

Center of pressure $(\mathrm{CoP})$ displacement signals in the medio-lateral direction were filtered digitally by a Butterworth low-pass filter with a $12 \mathrm{~Hz}$ cut-off frequency. Regarding the spatial magnitude of sway, Root Mean Square (RMS) and mean velocity (MV) in medio-lateral direction were calculated using equations proposed previously (Prieto, Myklebust, Hoffmann, Lovett, \& Myklebust, 1996). The RMS represents the average distance of the CoP regarding the " 0 " value (in this case); the higher the RMS, the larger the distance and the lower the performance in the task. The MV represents the neuromuscular activity needed to perform the task; in the present case, the higher the MV, the larger the neuromuscular activity (Paillard \& Noé, 2015). Moreover, we assessed the temporal dynamics of sway using detrended fluctuation analysis (DFA). Specifically, the $\alpha$-scaling exponent in medio-lateral direction was computed. This variable provides information about the long-range autocorrelation in the signal (Lin, Seol, Nussbaum, \& Madigan, 2008); it is related to the amount of information exchange between the participant and the environment. In the present case, the higher the $\alpha$-scaling, the more predictable (repetitive) pattern used to maintain the balance (Hausdorff, 2007).

SPSS software Version 21 (SPSS Inc., Chicago, IL, USA) was used for the statistical analysis. Parametric tests were used, as the Kolmogorov-Smirnov test verified that all variables met with the normality assumption. The mean and standard deviation were obtained by standard statistical methods. A mixed model MANOVA (3 Feedback [100\%, 67\%, 0\%] ×3 Test [Pre, Post, Retention]) was applied to determine the effect of feedback group and practice on the postural control. The follow up of the multivariate analysis was performed by means of univariate contrast. Pairwise comparisons with Bonferroni correction were requested when significant univariate contrasts were found. Partial eta-squared $\left(\eta_{\mathrm{p}}^{2}\right)$ values below .01, .01-.06, .06-.14, and above .14 were considered to have trivial, small, medium, and large effect sizes, respectively (Cohen, 1988). A $p$-value of .05 was accepted as the level of significance in all the statistical analyses.

\section{Results}

Multivariate analysis did not show differences between feedback groups in the RMS. A main effect of the feedback group on $\mathrm{MV}\left(F_{(2,27)}=3.25 ; p=.05 ; \eta_{\mathrm{p}}^{2}=.19\right)$ and $\alpha$-scaling exponent $\left(F_{(2,27)}=6.54 ; p=.005 ; \eta_{\mathrm{p}}^{2}=.33\right)$ was found. Pairwise comparisons revealed that $\alpha$-scaling exponent was higher in the $100 \%$ feedback 
group $(M=1.62, S D=0.03)$ than in the $0 \%$ feedback group $(M=1.48, S D=$ $0.03)(p<.05)$.

Moreover, there was a main effect of test in the RMS $\left(F_{(2,54)}=9.94 ; p<.001\right.$; $\left.\eta_{\mathrm{p}}^{2}=.27\right), \mathrm{MV}\left(F_{(2,54)}=9.28 ; p<.001 ; \eta_{\mathrm{p}}^{2}=.26\right)$ and $\alpha$-scaling exponent $\left(F_{(2,54)}=\right.$ $\left.4.67 ; p=.013 ; \eta_{\mathrm{p}}^{2}=.15\right)$. Pairwise comparisons revealed that RMS was higher in the pre-test $(M=57.44, S D=11.46)$ than in the post-test $(M=49.23, S D=9.44)$ $(p<.05)$. Moreover, RMS and MV were lower in the post-test (for MV $M=243.21$, $S D=63.08$ ) than in retention (for RMS $M=52.91, S D=10.50$ and for MV $M=276.68, S D=74.12$, respectively) $(p<.05)$.

Finally, there was an interaction effect of feedback group $\times$ test on $\mathrm{MV}\left(F_{(4,54)}\right.$ $\left.=3.51 ; p=.013 ; \eta_{\mathrm{p}}^{2}=.21\right)$ and $\alpha$-scaling exponent $\left(F_{(4,54)}=3.9 ; p=.007\right.$; $\left.\eta_{\mathrm{p}}^{2}=.22\right)$. The pairwise comparisons regarding this interaction effect are displayed in Figure 1. For the $100 \%$ and $67 \%$ feedback groups, MV was lower in the post-test ( $M=220.01, S D=40.96$ and $M=222.89, S D=46.68$, respectively) than in the retention (for $100 \%$ group $M=246.26, S D=36.14$ and for $67 \%$ group $M=262.48$, $S D=51.47)(p<.03)$. For the $0 \%$ feedback group, the MV was lower in the pre-test $(M=269.69, S D=45.72)$ and the post-test $(M=286.72, S D=76.19)$ than in the retention $(M=321.30, S D=101.40)(p<.01)$; also, for the $\alpha$-scaling exponent was higher in pre-test $(M=1.56, S D=0.08)$ and post-test $(M=1.45, S D=0.14)$ than in the retention $(M=1.44, S D=0.17)(p<.01)$. In the post-test, for both $(100 \%$ and $67 \%$ ) feedback groups, lower MV and higher $\alpha$-scaling exponent (for $100 \%$ and $67 \% \alpha$-scaling was $M=1.63, S D=0.45$ and $M=1.58, S D=0.12$, respectively) were found than in the $0 \%$ feedback group $(p<.05)$. In retention, the $100 \%$ feedback group had a higher $\alpha$-scaling exponent $(M=1.61, S D=0.47)$ than the $0 \%$ feedback group $(p<.01)$.

\section{Discussion}

The current study aimed to clarify whether a high frequency of verbal-terminalaugmented feedback $(100 \%)$ in the form of KP in adolescents would be beneficial for learning a relatively complex motor skill compared to a lower KP frequency (67\%) or no feedback (Sullivan et al., 2008; Wulf et al., 2010). Based on the fact that adults and children have differences in cognitive abilities (Sullivan et al., 2008) possibly impacting how feedback frequency will influence motor skill learning, it was hypothesized that providing feedback for every trial when adolescents are practicing motor tasks, especially for relatively complex skills such as that used in the present work, might influence positively motor skill learning (Sullivan et al., 2008; Wulf et al., 2010). Our results partially confirmed this hypothesis in terms of balance control patterns; that is, in pretest, for a similar performance (RMS), similar neuromuscular activity (MV) and balance control pattern ( $\alpha$-scaling exponent) were exhibited. Following practice, for a similar performance those who received feedback (100\% and 67\%) exhibited mainly less neuromuscular activity and balance control pattern than those in the $0 \%$ feedback group.

When children's performance is assessed during a dynamic balance task, the results revealed similar dynamic balance displacement (RMS) in the pre-test, the post-test, and the retention test, independent of the frequency of feedback provided. Our results support those found recently in older people wherein the concurrent 

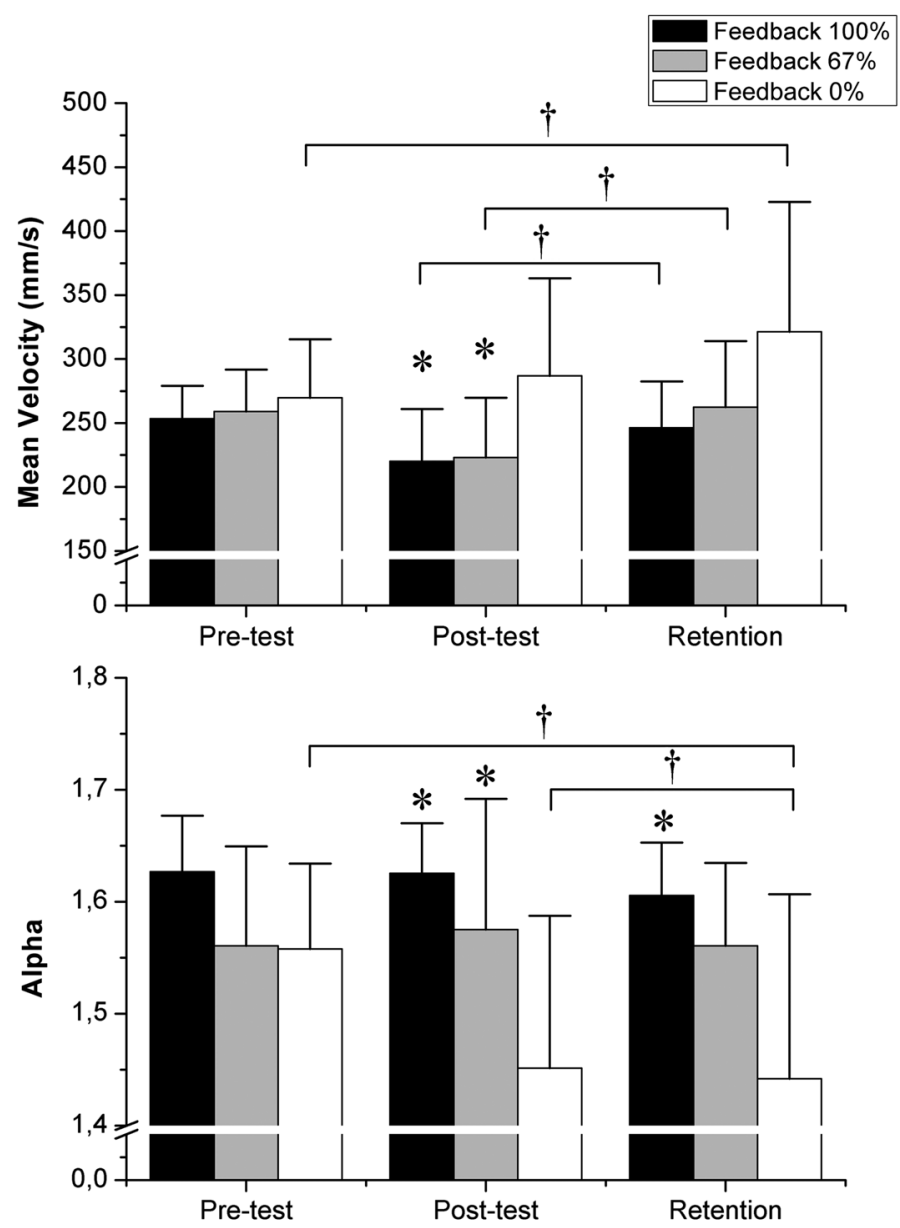

Figure 1 - Adolescents' balance performance according to the feedback group and test. Black columns refer to $100 \%$ feedback group; grey columns refer to $67 \%$ feedbacks group; white columns refer to $0 \%$ feedback group. *Significant differences with $0 \%$ feedback group. $\nmid$ Significant differences between types of test in a specific group.

augmented feedback does not enhance motor learning in a reactive balance task (Mansfield et al., 2017). However, this is contrary to Sullivan et al. (2008) and Wulf et al. (2010) who found higher task performance for children receiving augmented feedback more frequently. The reason why the current study failed to reveal improvement in the dynamic balance performance might be related to the type of verbal cues (external and internal in relation to the participant's body) used in this experiment. Considerable research (Palmer, Matsuyama, Irwin, Porter, \& Robinson, 2017; Wulf et al., 2010) has supported the claim that an external focus of attention results in superior learning. The use of cues in the present study that were 
internal in nature (e.g., focus on the movement of limbs) may have reduced the potency of the KP.

When performance is analyzed in terms of active control (i.e., MV) or how much effort to maintain balance is required, the children who did not receive feedback exhibited higher active control in the post-test than those who received more frequent feedback. As a result, receiving feedback seems to allow the children to maintain similar performance with lower active control in the retention than in the post-test (Fujii et al., 2016). A higher MV implies more postural readjustments to maintain similar dynamic balance performance is required. The no feedback group showed higher active control in the retention than in the pretest, that means when no feedback is reported children required a higher effort than those receiving feedback in new practices after a training session. For dynamic balance learning, these data suggest that PE teachers or instructors provide frequent feedback to students to help maintain similar performance with less postural readjustments.

In relation to the balance control patterns (measured by the $\alpha$-scaling), children who received feedback $(100 \%$ and $67 \%)$ presented a more stable pattern in the post-test than the control group. Moreover, only those who received $100 \%$ feedback still maintained a higher balance control pattern in retention than the $0 \%$ feedback group. Contrary to the influential guidance hypothesis (Schmidt et al., 1989) that postulates it is better to give less augmented feedback during acquisition so that students can use their own inherent feedback (Fujii et al., 2016), in the current study, when feedback was reported participants seemed to use this extra information precisely; that is, they focused on the cues provided instead of, for instance, any other information from the environment around (Coubard et al., 2014). Furthermore, when no feedback was provided, individuals were not able to maintain the balance control pattern. It seems that for adolescents, it would be better to provide feedback after every trial because, in comparison to those receiving less frequent feedback or no feedback, it helps to maintain the balance pattern not only during the practice session but during delayed retention tests.

The current study was conducted in two sessions wherein adolescents had 12 training trials, and a total of 21 trials across 24 hours. It is possible that only one training session is not enough to assess dynamic balance acquisition (i.e., a continuous task with high number of degrees of freedom). It should be noted, however, that the majority of the studies involving terminal feedback in the motor learning field are based on a single factor manipulation (i.e., the number of training sessions, the frequency of feedback, or the age of participants) with high risk of bias (Sattelmayer et al., 2016). Nonetheless, it is suggested that future studies consider the impact of including several training sessions with different frequencies of feedback to individuals for motor learning in terms of acquisition of dynamic balance.

In conclusion, adolescents who receive verbal-terminal-augmented KP after each trial $(100 \%)$ maintained a more stable balance control pattern, indicative of superior postural control while exhibiting similar dynamic balance displacement. Thus, adolescents who received high feedback frequencies (100\% and 67\%) required less effort and less postural readjustments than when no feedback is received to achieve comparable balance performance. 


\section{References}

Cohen, J. (1988). Statistical power analysis for the behavioral sciences (2nd ed.). Hillsdale, NJ: Lawrence Erlbaum.

Condon, C., \& Cremin, K. (2014). Static balance norms in children. Physiotherapy Research International: The Journal for Researchers and Clinicians in Physical Therapy, 19(1), 1-7. doi:10.1002/pri.1549

Coubard, O.A., Ferrufino, L., Nonaka, T., Zelada, O., Bril, B., \& Dietrich, G. (2014). One month of contemporary dance modulates fractal posture in aging. Frontiers in Aging Neuroscience, 6, 17. PubMed ID: 24611047 doi:10.3389/fnagi.2014.00017

Ferguson, A.N., \& Bowey, J.A. (2005). Global processing speed as a mediator of developmental changes in children's auditory memory span. Journal of Experimental Child Psychology, 91(2), 89-112. PubMed ID: 15890172 doi:10.1016/j.jecp.2004. 12.006

Fujii, S., Lulic, T., \& Chen, J.L. (2016). More feedback is better than less: Learning a novel upper limb joint coordination pattern with augmented auditory feedback. Frontiers in Neuroscience, 10, 251. PubMed ID: 27375414 doi:10.3389/fnins.2016.00251

Guadagnoli, M.A., \& Lee, T.D. (2004). Challenge point: A framework for conceptualizing the effects of various practice conditions in motor learning. Journal of Motor Behavior, 36(2), 212-224. PubMed ID: 15130871 doi:10.3200/JMBR.36.2.212-224

Hausdorff, J.M. (2007). Gait dynamics, fractals and falls: Finding meaning in the stride-tostride fluctuations of human walking. Human movement science, 26(4), 555-589. PubMed ID: 17618701 doi:10.1016/j.humov.2007.05.003

Landin, D. (1994). The role of verbal cues in skill learning. Quest, 46(3), 299-313. doi:10. 1080/00336297.1994.10484128

Lin, D., Seol, H., Nussbaum, M.A., \& Madigan, M.L. (2008). Reliability of COP-based postural sway measures and age-related differences. Gait \& Posture, 28(2), 337-342. PubMed ID: 18316191 doi:10.1016/j.gaitpost.2008.01.005

Magill, R.A. (1998). 1997 C. H. McCloy Research Lecture: Knowledge is more than we can talk about: Implicit learning in motor skill acquisition. Research Quarterly for Exercise and Sport, 69(2), 104-110. PubMed ID: 9635325 doi:10.1080/02701367. 1998.10607676

Magill, R.A. (2001). Motor learning: Concepts and applications (6th ed.). Boston, MA: McGraw-Hill.

Mansfield, A., Aqui, A., Fraser, J.E., Rajachandrakumar, R., Lakhani, B., \& Patterson, K.K. (2017). Can augmented feedback facilitate learning a reactive balance task among older adults? Experimental Brain Research, 235(1), 293-304. PubMed ID: 27709269 doi:10.1007/s00221-016-4790-6

Paillard, T., \& Noé, F. (2015). Techniques and Methods for Testing the Postural Function in Healthy and Pathological Subjects. BioMed Research International, 2015. doi:10. $1155 / 2015 / 891390$

Palmer, K.K., Matsuyama, A.L., Irwin, J.M., Porter, J.M., \& Robinson, L.E. (2017). The effect of attentional focus cues on object control performance in elementary children. Physical Education and Sport Pedagogy, 22(6), 580-588. doi:10.1080/17408989. 2017.1294667

Perreault, M.E., \& French, K.E. (2016). Differences in children's thinking and learning during attentional focus instruction. Human Movement Science, 45, 154-160. PubMed ID: 26638048 doi:10.1016/j.humov.2015.11.013

Pollock, B.J., \& Lee, T.D. (1997). Dissociated contextual interference effects in children and adults. Perceptual and Motor Skills, 84(3 Pt 1), 851-858. PubMed ID: 9172193 doi:10. 2466/pms.1997.84.3.851 
Prieto, T.E., Myklebust, J.B., Hoffmann, R.G., Lovett, E.G., \& Myklebust, B.M. (1996). Measures of postural steadiness: Differences between healthy young and elderly adults. IEEE Transactions on Bio-Medical Engineering, 43(9), 956-966. PubMed ID: 9214811 doi: $10.1109 / 10.532130$

Rink, J. (2006). Teaching physical education for learning (5th ed.). Boston, MA: McGrawHill.

Sattelmayer, M., Elsig, S., Hilfiker, R., \& Baer, G. (2016). A systematic review and metaanalysis of selected motor learning principles in physiotherapy and medical education. BMC Medical Education, 16, 15. doi:10.1186/s12909-016-0538-z

Schmidt, R., \& Lee, T. (2011). Motor control and learning: A behavioral emphasis (5th ed.). Champaign, IL: Human Kinetics.

Schmidt, R.A., \& Wrisberg, C.A. (2008). Motor learning and performance: A situationbased learning approach (4th ed.). Champaign, IL: Human Kinetics.

Schmidt, R.A., Young, D.E., Swinnen, S., \& Shapiro, D.C. (1989). Summary knowledge of results for skill acquisition: Support for the guidance hypothesis. Journal of Experimental Psychology. Learning, Memory, and Cognition, 15(2), 352-359. PubMed ID: 2522520 doi:10.1037/0278-7393.15.2.352

Sharma, D.A., Chevidikunnan, M.F., Khan, F.R., \& Gaowgzeh, R.A. (2016). Effectiveness of knowledge of result and knowledge of performance in the learning of a skilled motor activity by healthy young adults. Journal of Physical Therapy Science, 28(5), 14821486. PubMed ID: 27313355 doi:10.1589/jpts.28.1482

Sigrist, R., Rauter, G., Riener, R., \& Wolf, P. (2013). Augmented visual, auditory, haptic, and multimodal feedback in motor learning: A review. Psychonomic Bulletin \& Review, 20(1), 21-53. PubMed ID: 23132605 doi:10.3758/s13423-012-0333-8

Sullivan, K.J., Kantak, S.S., \& Burtner, P.A. (2008). Motor learning in children: Feedback effects on skill acquisition. Physical Therapy, 88(6), 720-732. PubMed ID: 18339797 doi: $10.2522 / \mathrm{ptj} .20070196$

Sülzenbrück, S., \& Heuer, H. (2011). Type of visual feedback during practice influences the precision of the acquired internal model of a complex visuo-motor transformation. Ergonomics, 54(1), 34-46. PubMed ID: 21181587 doi:10.1080/00140139.2010. 535023

Thomas, J.R., Cotten, D.J., Spieth, W.R., \& Abraham, N.L. (1975). Effects of fatigue on stabilometer performance and learning of males and females. Medicine and science in sports, 7(3), 203-206.

Villarrasa-Sapiña, I., García-Massó, X., Serra-Añó, P., Garcia-Lucerga, C., Gonzalez, L.-M., \& Lurbe, E. (2016). Differences in intermittent postural control between normal-weight and obese children. Gait \& Posture, 49, 1-6. doi:10.1016/j.gaitpost. 2016.06.012

Wishart, L.R., \& Lee, T.D. (1997). Effects of aging and reduced relative frequency of knowledge of results on learning a motor skill. Perceptual and Motor Skills, 84, 11071122. doi:10.2466/pms.1997.84.3.1107

Wulf, G., Chiviacowsky, S., Schiller, E., \& Avila, L.T.G. (2010). Frequent external-focus feedback enhances motor learning. Frontiers in Psychology, 1, 190. PubMed ID: 21833250 doi:10.3389/fpsyg.2010.00190

Wulf, G., \& Schmidt, R.A. (1989). The learning of generalized motor programs: Reducing the relative frequency of knowledge of results enhances memory. Journal of Experimental Psychology: Learning, Memory, and Cognition, 15(4), 748-757. doi:10.1037/ 0278-7393.15.4.748

Wulf, G., \& Shea, C.H. (2002). Principles derived from the study of simple skills do not generalize to complex skill learning. Psychonomic Bulletin \& Review, 9(2), 185-211. PubMed ID: 12120783 doi:10.3758/BF03196276 
Wulf, G., \& Shea, C.H. (2004). Understanding the role of augmented feedback: The good, the bad and the ugly. In A.M. Williams\& N.J. Hodges (Eds.), Skill Acquisition in Sport (pp. 121-144). New York, NY: Routledge.

Wulf, G., Shea, C.H., \& Matschiner, S. (1998). Frequent feedback enhances complex motor skill learning. Journal of Motor Behavior, 30(2), 180-192. PubMed ID: 20037033 doi:10.1080/00222899809601335

Wulf, G., Weigelt, M., Poulter, D., \& McNevin, N. (2003). Attentional focus on suprapostural tasks affects balance learning. The Quarterly Journal of Experimental Psychology. A, Human Experimental Psychology, 56(7), 1191-1211. PubMed ID: 12959910 doi: $10.1080 / 02724980343000062$ 